\title{
The Value of Digital Subtraction Angiography in Interlaminar Cervical Epidural Steroid Injections
}

\author{
Anand Prem, MD*, Suwarna Anand, MD and Arvind Chandrashekar, MD \\ University of Mississippi Medical Center, Jackson, MS, USA
}

\section{Introduction}

Neck and upper extremity radicular pain is a common indication for ambulatory clinic visits for Chronic pain. By the time a patient presents to a Pain specialist, they have often exhausted all conservative treatment modalities including rest, heat, Nonsteroidal anti-inflammatory drugs, muscle relaxants and Physical therapy. These patients have often failed a trial of opioids as well and deemed not a candidate for surgery by a Spine surgeon. Imaging studies may reveal Cervical degenerative disc disease, neural foraminal stenosis or central canal stenosis. After a trial of Gabapentin and tricyclic antidepressants it is reasonable to consider a trial of interventional treatment options. Cervical Epidural Steroid injections are among the commonest procedures in Interventional Pain clinics to treat Cervical Radiculopathy with good evidence for short term benefit in these cases. While performance of this procedure under fluoroscopic guidance has significantly improved the safety of the procedure, rare but catastrophic complications can still occur either from direct spinal cord injury or if particulate steroids are injected into the radicular spinal arteries leading to embolism of the anterior or posterior spinal arteries and subsequent cord infarction. Digital Subtraction Angiography (DSA) while recommended particularly for Cervical transforaminal injections, is not widely used in interlaminar epidural steroid injections, though it provides vital information to avoid devastating neurological complications. We present a case where digital subtraction angiography helped us avoid the potential complications of steroids injected intravascularly during a fluoroscopically guided Cervical Interlaminar Epidural Steroid injection.

\section{Materials and Methods}

No IRB approval required as all patient identifiers have been removed.

Informed consent obtained from patient for this report.

\section{Case Report}

36-year-old Caucasian male presented to our Pain Clinic with a 3-year history of Chronic neck and Right arm pain suggestive of Cervical radiculopathy. Examination was positive for Spurling's sign and diminished triceps reflex on the Right. There was no obvious sensory or motor deficit. MRI Cervical
Spine revealed multilevel disc degeneration, disc osteophyte complexes with neural foraminal narrowing worst at Right C5-6 and C6-7. Patient had 2 prior Cervical Epidural steroid injections in the past year with $70 \%$ pain relief lasting 4-5 months. As radicular symptoms had recurred, he was brought back for a repeat Cervical Epidural steroid injection. After a thorough physical examination to confirm previous findings, an informed consent was obtained for the planned Cervical Epidural Steroid injection under fluoroscopic guidance. Under strict asepsis, the Cervical epidural space was accessed under fluoroscopic guidance with a 20G Tuohy needle via Right interlaminar approach at the C7-T1 interspace using loss of resistance to saline technique without any paresthesia. Aspiration was grossly negative for CSF or blood. Needle position in the epidural space was appropriate in anteroposterior and lateral fluoroscopic views. Digital subtraction angiography with $1 \mathrm{ml}$ of Isovue 300M contrast dye however showed prominent intravascular spread of dye in the AP view. The needle was removed and redirected slightly medially into the epidural space without incident. Repeat DSA showed absent vascular spread and typical epidural dye spread in both AP and lateral views. $6 \mathrm{mg}$ of Betamethasone with $1 \mathrm{ml}$ of $0.1 \%$ bupivacaine and $2 \mathrm{ml}$ of saline was injected into the epidural space. Patient tolerated the procedure well without complications. Patient was examined post-procedure and demonstrated significantly improved pain in the Right Upper extremity and neck with increased range of motion of the neck and no motor or sensory deficit. On follow up, patient had greater than $80 \%$ improvement in pain that lasted about 4 months.

\section{Discussion}

Cervical Epidural steroid injections are widely used to treat neck pain with radiculopathy. While complications are

*Corresponding author: Anand Prem, MD, Associate Professor, Anesthesiology, Medical Director, University Pain Clinic, University of Mississippi Medical Center, 2500 N State Street, S-108, Jackson, MS 39216, USA, Tel: (601)-951-3791

Accepted: August 29, 2020

Published online: August 31, 2020

Citation: Prem A, Anand S, Chandrashekar A (2020) The Value of Digital Subtraction Angiography in Interlaminar Cervical Epidural Steroid Injections. J Clin Anesth Pain Manag 4(2):129-130 
rare, data from Closed Claims Analysis shows most pain treatment claims are related to Epidural steroid injections and facet blocks. Devastating neurological complications from Cervical Epidural steroid injections, especially transforaminal injections occur with injection of particulate steroids into a radiculo-medullary or spinal-medullary artery causing embolization and infarction of the spinal cord [1]. An expert panel convened under the FDA Safe Use Initiative made recommendations in 2015 based on available evidence to make these injections safer including the avoidance of particulate steroids along with specific anatomical considerations for cervical injections and use of fluoroscopy [1,2]. In a meta-analysis by Visnjevac, et al. published in 2015 comparing Digital Subtraction Angiography to Real time Fluoroscopy, there was a $32 \%$ improvement in the detection of an intravascular injection by DSA that was missed by real time fluoroscopy alone. This study raised the alarming potential of missing about $30 \%$ of intravascular needle tip placements during Cervical Epidural steroid injections done using Real time fluoroscopy alone [3]. However, the reported complication rate during Cervical Epidural steroid injections in current literature is less than $1 \%$. The need for DSA routinely however was not widely accepted citing poor availability, significantly increased cost, and increased radiation exposure [4]. While live fluoroscopy during contrast injection may be beneficial, the fleeting image of vascular uptake is often missed. In our case, DSA was the only positive indicator of vascular uptake requiring needle repositioning, avoiding the potentially devastating complications of intravascular particulate steroid injection. Efficacy of the procedure in terms of pain relief had the steroids unintentionally been injected intravascularly would also have been compromised without DSA, making a case for its routine use in CESI.

This case along with the recommendations of the Multidisciplinary Workgroup brought about a change in our practice to using only non-particulate steroids such as Dexamethasone and DSA in all Cervical Epidural Steroid injections. While these procedures are largely safe in well trained hands, considering the low rate of reported complications, as Interventional Pain Physicians it is imperative for us to take all possible measures to minimize the risk of patient harm while providing optimal pain relief. This reiterated the need for further study of the feasibility of making DSA the gold standard for all Cervical Epidural steroid injections. Above all, "Do no harm".

\section{Funding}

None.

\section{Disclosures}

None.

\section{Comment}

Consent for this report obtained from all authors noted above.

\section{References}

1. James P Rathmell, Honorio T Benzon, Paul Dreyfuss, et al. (2015) Safeguards to prevent neurologic complications after epidural steroid injections: Consensus opinions from a multidisciplinary working group and national organizations. Anesthesiology 122: 974-984.

2. Abrams SE, O'Connor TC (1996) Complications associated with epidural steroid injections. Reg Anesth 21: 149-162.

3. Visnjevac O, Kim P, Farid-Davari S, et al. (2015) Digital subtraction angiography versus real-time fluoroscopy for detection of intravascular injections: Meta-analysis of prospective studies. Pain Physician 18: 29-36.

4. Laxmaiah Manchikanti, Frank Falco (2015) Safeguards to prevent neurologic complications after epidural steroid injections: Analysis of evidence and lack of applicability of controversial policies. Pain Physician 18: E129-E138.

DOI: $10.36959 / 377 / 342$ 